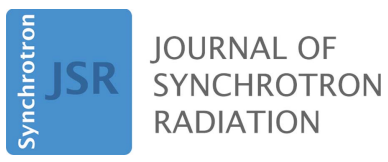

ISSN 1600-5775

Received 22 March 2019

Accepted 28 June 2019

Edited by G. Grübel, HASYLAB at DESY, Germany

Keywords: serial crystallography; sample delivery; fixed targets; acoustic dispensing.
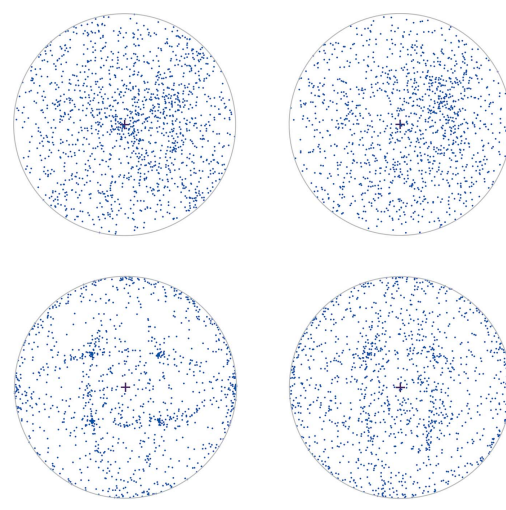

OPEN $\odot$ ACCESS

\section{Reducing sample consumption for serial crystallography using acoustic drop ejection}

Bradley Davy, a Danny Axford, ' John H. Beale, ${ }^{a}$ Agata Butryn, ${ }^{a}$ Peter Docker, Ali Ebrahim, ${ }^{b, a}$ Gabriel Leen, ${ }^{c, d}$ Allen M. Orville, ${ }^{a, e}$ Robin L. Owen $^{\text {a* }}$ and Pierre Aller ${ }^{\mathrm{a} *}$

${ }^{\mathbf{a}}$ Diamond Light Source, Harwell Science and Innovation Campus, Didcot, Oxfordshire OX11 ODE, UK, ${ }^{\mathbf{b}}$ School of Biological Sciences, University of Essex, Wivenhoe Park, Colchester, Essex CO4 3SQ, UK, 'PolyPico Technologies Ltd, Unit 10, Airways Technology Park, Rathmacullig Wes, Ballygarvan, Cork T12 DY95, Ireland, ${ }^{\mathbf{d} D e p a r t m e n t}$ of Electronic and Computer Engineering, University of Limerick, Ireland, and ${ }^{\mathbf{e}}$ Research Complex at Harwell, Rutherford Appleton Laboratory, Didcot OX11 OFA, UK. *Correspondence e-mail: robin.owen@diamond.ac.uk, pierre.aller@diamond.ac.uk

Efficient sample delivery is an essential aspect of serial crystallography at both synchrotrons and X-ray free-electron lasers. Rastering fixed target chips through the X-ray beam is an efficient method for serial delivery from the perspectives of both sample consumption and beam time usage. Here, an approach for loading fixed targets using acoustic drop ejection is presented that does not compromise crystal quality, can reduce sample consumption by more than an order of magnitude and allows serial diffraction to be collected from a larger proportion of the crystals in the slurry.

\section{Introduction}

Serial femtosecond crystallography (SFX) at X-ray free-electron lasers (XFELs) has become an important facet of the crystallographers' toolbox, allowing both time-resolved and ground-state measurements of X-ray sensitive samples (Schlichting, 2015). However, the opportunities that the high peak brilliance and femtosecond duration of XFEL pulses provide come with a challenge: the need to provide new samples at the repetition rate of the X-ray source or detector. Several approaches have been developed to meet this challenge ranging from liquid jets to high viscosity extruders, ondemand droplet injectors coupled to a tape drive, and fixed targets (Grünbein \& Kovacs, 2019; Martiel et al., 2019). This complementary range of delivery solutions means that an approach can be chosen and tailored to best suit the experiment at hand.

The success and impact of SFX has inspired the development and implementation of serial synchrotron crystallography (SSX), where many of the same sample delivery techniques are used (Diederichs \& Wang, 2017). The subsequent success of SSX has now driven the development of synchrotron beamlines dedicated to serial crystallography, such as P14.EH2 at PETRA III (http://www.embl-hamburg.de/ services/mx/P14_EH2/index.html), and this illustrates the desire of structural biologists to exploit serial approaches.

A challenge common to many serial approaches is sample consumption. The volume of sample consumed is many orders of magnitude greater than that required for traditional synchrotron approaches when a complete dataset may be obtained from a single crystal held at $100 \mathrm{~K}$. Indeed, the sample requirements for a serial experiment are often an unwelcome surprise for the first-time user of SFX or SSX, as usually only a single 'still' image is collected from each crystal. 
This is reflected by developments to reduce sample consumption for serial experiments such as flow-focusing for inflow SSX (Monteiro et al., 2019).

Recently, we have developed fixed target sample delivery as a serial approach that works well at both synchrotrons and XFELs. These are based on thin films (Doak et al., 2018) and, predominantly for us, silicon nitride 'chips' (Ebrahim et al., 2019). Typically, to load a silicon nitride chip, $\sim 100-200 \mu \mathrm{l}$ of crystal slurry is required. This slurry is pipetted over a chip and crystals are drawn to the apertures through use of a weak vacuum. Sufficient data for structure solution can typically be obtained from a single chip.

Acoustic dispensing is a technique that uses high-frequency acoustic waves to dispense small volumes of liquid. The ejected droplets may contain protein crystals (Soares et al., 2011; Roessler et al., 2016; Fuller et al., 2017), live cells (Demirci \& Montesano, 2007) or indeed almost any small molecule (Teplitsky et al., 2015). Commonly referred to as acoustic drop ejection (ADE), here we use a variant that makes use of disposable dispensing cartridges allowing rapid switching between samples (Leen, 2016). Using the commercially available PolyPico pico-litre dispenser (https://www. polypico.com) synchronized with compact, high-precision $x y z$ stages (http://www.smaract.com), we demonstrate the use of ADE to dramatically reduce sample consumption for fixed target serial crystallography.

\section{Methods}

The loading of fixed targets using acoustic dispensing is a twostep process with a calibration step required prior to chip loading. For convenience we physically separate these steps [Figs. 1(a) and 1(b)]. The same PolyPico head dispenser was used for both aspects of the experiment and was mounted on kinematic mounts allowing transfer between calibration and loading in a few seconds.

\subsection{Drop calibration}

For optimal loading of chips, the volume of droplets ejected by the PolyPico dispenser should be calibrated for each crystal slurry. Using a pipette and a tip-like adapter, the crystal slurry

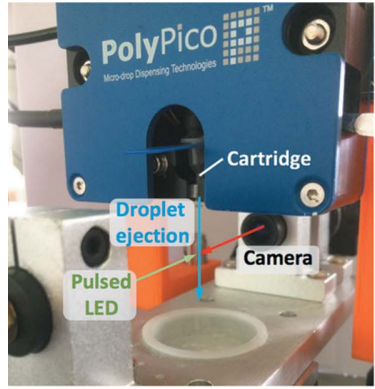

(a)

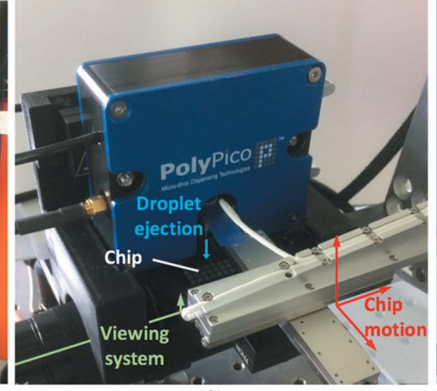

(b)

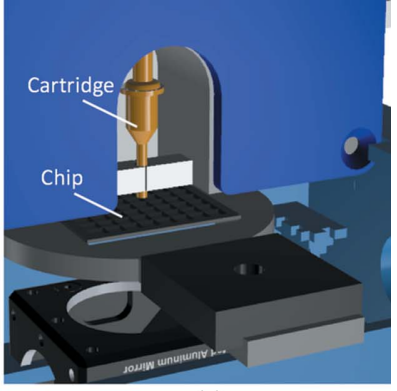

(c)
Figure 1

Experimental setup for $(a)$ calibration of ejected droplets and $(b)$ chip loading; in each, the direction of droplet ejection is shown in blue. (c) Schematic of chip loading from a similar viewpoint to $(b)$ with translation stages hidden and the cartridge highlighted in yellow. is loaded into a cartridge which has a dispensing aperture ranging from $30 \mu \mathrm{m}$ to $150 \mu \mathrm{m}$ in diameter. For the experiments described here, we loaded 10-20 $\mu \mathrm{l}$ of slurry into cartridges with any slurry not used easily recovered after the experiment using the same pipette and adapter. The cartridge aperture size is chosen based on the typical size of the crystals in the slurry. In practice, we find that an aperture diameter approximately twice the size of the crystals used works well as a compromise between minimizing drop size and avoiding clogging if larger crystals are present. The width, amplitude and frequency of the acoustic wave applied to the cartridge base must be tuned until stable droplets are ejected from the crystal slurry. Ejected droplets are visualized using a highresolution camera and stroboscopic LED [Fig. 1(a)] with image recognition software allowing real-time readback of the average droplet volume. Typically, when using a $1 \mathrm{kHz}$ acoustic wave and a cartridge aperture of $100 \mu \mathrm{m}, 80-100 \mathrm{pl}$ (approximate diameter $60 \mu \mathrm{m}$ ) droplets can be obtained. Once the optimal parameters for ADE of crystal slurry have been determined, chips can be loaded.

\subsection{Chip loading}

The setup for ADE loading of fixed targets is shown in Figs. 1(b) and 1(c). Chips are mounted on a three-axis stage and can be viewed through a high-resolution camera which allows viewing of both fixed targets and droplets ejected by the dispensing head. The tip of the dispensing head is within $0.5 \mathrm{~mm}$ of the surface of the chip. Following alignment of chip fiducials, chips can be moved as previously described (Sherrell et al., 2015). In this case the stages act as the 'master', sending a TTL pulse to the dispensing head with droplets ejected on demand when each aperture is reached. Following the ejection of a user-defined number of droplets at $1 \mathrm{kHz}$, the stages move to the next aperture on the chip. The loading of a chip with 25600 positions takes less than 4 min and consumes less than $4 \mu \mathrm{l}$ of slurry. To avoid dehydration, the chip and dispensing head are enclosed in a high-relative-humidity environment $(>90 \%)$ [Fig. 1(b)]. Following loading, the chips are sealed with a thin film (typically $6 \mu \mathrm{m}$ ) of mylar. Chips with a funnelshaped aperture (size of the small end of the funnel: $7 \mu \mathrm{m}$ ) were used; the volume of each aperture was $\sim 160 \mathrm{pl}$, and apertures are spaced by $125 \mu \mathrm{m}$ (centre-of-aperture to centre-of-aperture distance).

In order to conserve sample and also minimize the beam time required for $\mathrm{X}$-ray data collection, only the central area of chips was acoustically loaded (6×6 'city blocks', 14400 apertures) in the experiments described here. In this case, the time required to load a chip was 2 min $15 \mathrm{~s}$. In total, the complete acoustic loading process including alignment and loading takes approximately 5 min (full chip), and throughput is equal to or faster than X-ray data 
collection. Chips were loaded by both using the ADE approach described above and also, for comparison, manually using a pipette.

\subsection{Sample preparation}

Microcrystals of chicken egg-white lysozyme (HEWL) were prepared using an adaptation of a previously described protocol. In brief, high-purity lysozyme powder (SigmaAldrich L6876-5 G) was resuspended in $100 \mathrm{~m} M$ sodium acetate $\mathrm{pH} 3.0$ to a final concentration of $25 \mathrm{mg} \mathrm{ml}^{-1}$ and mixed with an equal volume of crystallization buffer $(16.8 \%$ $w / v$ sodium chloride, $4.8 \% w / v$ PEG 6000 and $0.06 M$ sodium acetate $\mathrm{pH} 3.0$ ) at room temperature. The mixture was vortexed for $10 \mathrm{~s}$ and then left for an hour until crystal growth saturation. Using this method, we obtained homogeneous rectangular crystals with an average size of $10 \mu \mathrm{m} \times 10 \mu \mathrm{m} \times$ $15 \mu \mathrm{m}$. Microcrystals of copper nitrite reductase from Achromobacter cycloclastes (AcNiR) ranging in size from $15 \mu \mathrm{m}$ to $70 \mu \mathrm{m}$ were grown using a protocol described previously (Ebrahim et al., 2019). The concentration of crystals in each slurry was estimated using a Hemocytometer cell counter.

\subsection{X-ray data collection}

Following loading, chips were transferred to Beamline I24, Diamond Light Source. Diffraction data were collected as previously described (Owen et al., 2017) though only from the central region of chips loaded by the PolyPico (14400 apertures), with data collection taking $4 \mathrm{~min} 20 \mathrm{~s}$. Data were collected using an X-ray energy of $12.8 \mathrm{keV}$, a beam size of $7 \mu \mathrm{m} \times 6 \mu \mathrm{m}, 10 \mathrm{~ms}$ exposures and a flux attenuated to $8 \times 10^{11}$ photons s $^{-1}$.

Hit-rates were obtained using dials.stills_process (Winter et al., 2018; Brewster et al., 2016, 2018) with up to ten lattices per image indexed. Subsequent scaling and merging of data was performed using PRIME (Uervirojnangkoorn et al., 2015). In all cases the majority of indexed images contained a single lattice with the percentage of single lattice images being $77 \%$ (HEWL, PolyPico loaded), 81\% (HEWL, pipette loaded), $85 \%$ ( $A c \mathrm{NiR}$ PolyPico loaded) and 66\% ( $A c \mathrm{NiR}$, pipette loaded). In the following, we define the diffraction hit-rate as the total number of indexed patterns divided by the number of collected images.

\section{Results}

In preparatory experiments, we varied and defined the optimal number of acoustically ejected droplets. When dispensing two drops per single chip aperture, we observed higher hit-rates than when using a single droplet. The dispensing of three or more drops overflowed the apertures resulting in excess liquid on the surface of the chip. Therefore, all of the results presented here were obtained using two droplets per aperture.

Diffraction hit-rates for HEWL crystals loaded manually and using acoustic dispensing as a function of crystal
Table 1

HEWL loading parameters and hit-rates.

The diffraction hit-rate (DHR) and absolute hit-rate (AHR) are defined in the text. Volumes dispensed $(V)$ for the PolyPico are an upper bound: droplet volumes vary from drop to drop so a conservative average value is used.

\begin{tabular}{llllcll}
\hline $\begin{array}{l}\text { Loading } \\
\text { method }\end{array}$ & $\left.\begin{array}{l}\text { Crystal } \\
\text { concentration } \\
(\text { crystals ml }\end{array}{ }^{-1}\right)$ & $\begin{array}{l}V \\
(\mu \mathrm{l})\end{array}$ & $\begin{array}{l}\text { Calculated } \\
\text { crystals } \\
\text { dispensed }\end{array}$ & $\begin{array}{l}\text { Indexed } \\
\text { patterns }\end{array}$ & $\begin{array}{l}\text { DHR } \\
(\%)\end{array}$ & $\begin{array}{l}\text { AHR } \\
(\%)\end{array}$ \\
\hline Pipette & $5 \times 10^{4}$ & 75 & 3750 & 2053 & 14.3 & 55 \\
Pipette & $1 \times 10^{5}$ & 75 & 7500 & 2129 & 14.8 & 28 \\
Pipette & $5 \times 10^{5}$ & 75 & 37500 & 4850 & 33.7 & 13 \\
Pipette & $1 \times 10^{6}$ & 75 & 75000 & 10462 & 72.6 & 14 \\
PolyPico & $5 \times 10^{5}$ & 3 & 1500 & 1311 & 9.1 & 87 \\
PolyPico & $1 \times 10^{6}$ & 3 & 3000 & 1763 & 12.2 & 59 \\
PolyPico & $1.5 \times 10^{6}$ & 3 & 4500 & 2883 & 20.0 & 64 \\
PolyPico & $2 \times 10^{6}$ & 3 & 6000 & 4573 & 31.8 & 76 \\
PolyPico & $2.5 \times 10^{6}$ & 3 & 7500 & 3355 & 23.3 & 45 \\
\hline
\end{tabular}

concentration are shown in Table 1. As might be expected, in both cases diffraction hit-rates increase with crystal concentration. Also, for a given concentration, higher diffraction hitrates are obtained using pipette loading. However, acoustic loading requires a significantly lower volume crystal slurry to achieve these, as illustrated by the number of diffraction hits obtained per dispensed microlitre of crystal slurry (Fig. 2).

Similar trends are seen for $A c$ NiR crystals (Fig. 2, Table 2), which significantly differ from HEWL crystals both in shape and chemical composition of the crystallization conditions, with an increasing hit-rate for both pipette and acoustically loaded chips as a function of slurry concentration. Higher diffraction hit-rates are also seen for pipette loaded chips at the expense of increased sample consumption.

Using the crystal concentration measured as described above, the number of crystals used in each experiment, and hence the fraction from which diffraction was recorded, can be estimated. We refer to this quantity as the absolute hit-rate

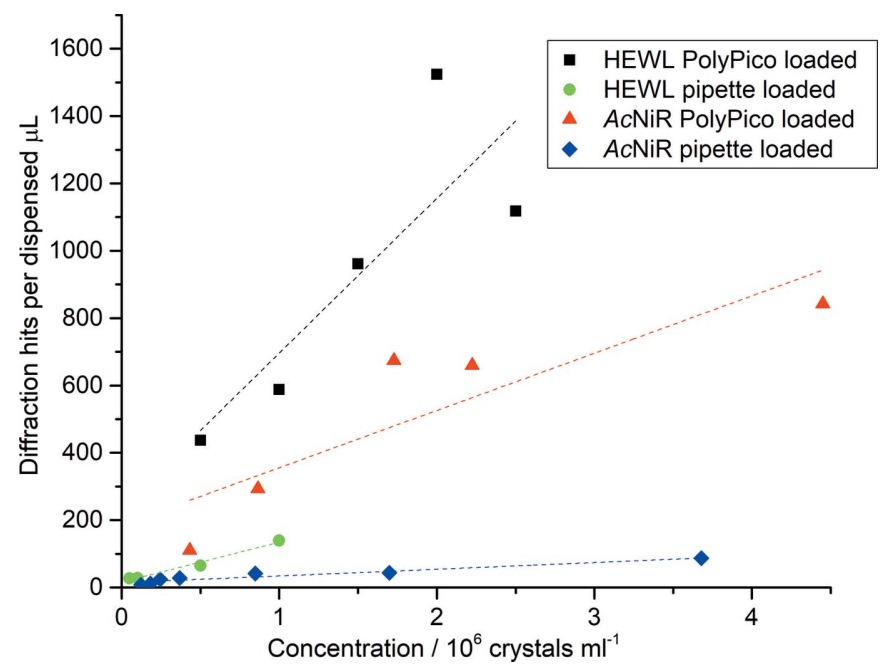

Figure 2

Diffraction hits per unit volume of dispensed crystal slurry. Acoustic dispensing results in more than a fivefold increase in hits per unit volume of slurry consumed at all concentrations for HEWL and a tenfold increase for $A c \mathrm{NiR}$. 
Table 2

$A c \mathrm{NiR}$ loading parameters and hit-rates.

The diffraction hit-rate (DHR) and absolute hit-rate (AHR) are defined in the text; $V$ is the volume dispensed. Note that for pipette-loaded $A c \mathrm{NiR}$, diffraction data were collected from a full chip (25 600 apertures) in contrast to all other data which were collected from 14400 apertures.

\begin{tabular}{llllrrr}
\hline $\begin{array}{l}\text { Loading } \\
\text { method }\end{array}$ & $\left.\begin{array}{l}\text { Crystal } \\
\text { concentration } \\
(\text { crystals ml }\end{array}{ }^{-1}\right)$ & $V(\mu \mathrm{l})$ & $\begin{array}{l}\text { Calculated } \\
\text { crystals } \\
\text { dispensed }\end{array}$ & $\begin{array}{l}\text { Indexed } \\
\text { patterns }\end{array}$ & $\begin{array}{l}\text { DHR } \\
(\%)\end{array}$ & $\begin{array}{l}\text { AHR } \\
(\%)\end{array}$ \\
\hline Pipette & $1.2 \times 10^{5}$ & 150 & 18000 & 1185 & 4.6 & 6.6 \\
Pipette & $1.8 \times 10^{5}$ & 150 & 27000 & 1687 & 6.6 & 6.2 \\
Pipette & $2.5 \times 10^{5}$ & 150 & 37500 & 3574 & 14.0 & 9.5 \\
Pipette & $3.7 \times 10^{5}$ & 150 & 55500 & 4145 & 16.2 & 7.5 \\
Pipette & $8.5 \times 10^{5}$ & 150 & 127500 & 6223 & 24.3 & 4.9 \\
Pipette & $1.7 \times 10^{6}$ & 150 & 255000 & 6587 & 25.7 & 2.6 \\
Pipette & $3.7 \times 10^{6}$ & 150 & 555000 & 13061 & 51.0 & 2.4 \\
PolyPico & $4.3 \times 10^{5}$ & 3 & 1290 & 332 & 2.3 & 25.7 \\
PolyPico & $8.6 \times 10^{5}$ & 3 & 2580 & 878 & 6.1 & 34.0 \\
PolyPico & $1.7 \times 10^{6}$ & 3 & 5100 & 2023 & 14.1 & 39.7 \\
PolyPico & $2.2 \times 10^{6}$ & 3 & 6600 & 1979 & 13.7 & 30.0 \\
PolyPico & $4.4 \times 10^{6}$ & 3 & 13200 & 2526 & 17.5 & 19.1 \\
\hline
\end{tabular}

and it is given for HEWL and $A c \mathrm{NiR}$ in Tables 1 and 2, respectively. For pipette loading, it can be seen that, although higher diffraction hit-rates are achieved by increasing the crystal slurry concentration (this could also be achieved by simply increasing the volume of slurry loaded onto the chip), this is at the expense of the absolute hit-rate, with diffraction recorded from a decreasing proportion of the crystals used in the loading process. Although the diffraction hit-rate may be lower for acoustically loaded fixed targets, a larger proportion of the crystals grown produce a diffraction pattern.

Importantly, the loading method does not significantly affect the quality of diffraction observed (Fig. 3). Both HEWL and $A c \mathrm{NiR}$ crystals exhibit similar $R_{\text {split }}$ and $\mathrm{CC}_{1 / 2}$ for both acoustic and pipette loading, and in all cases data quality is

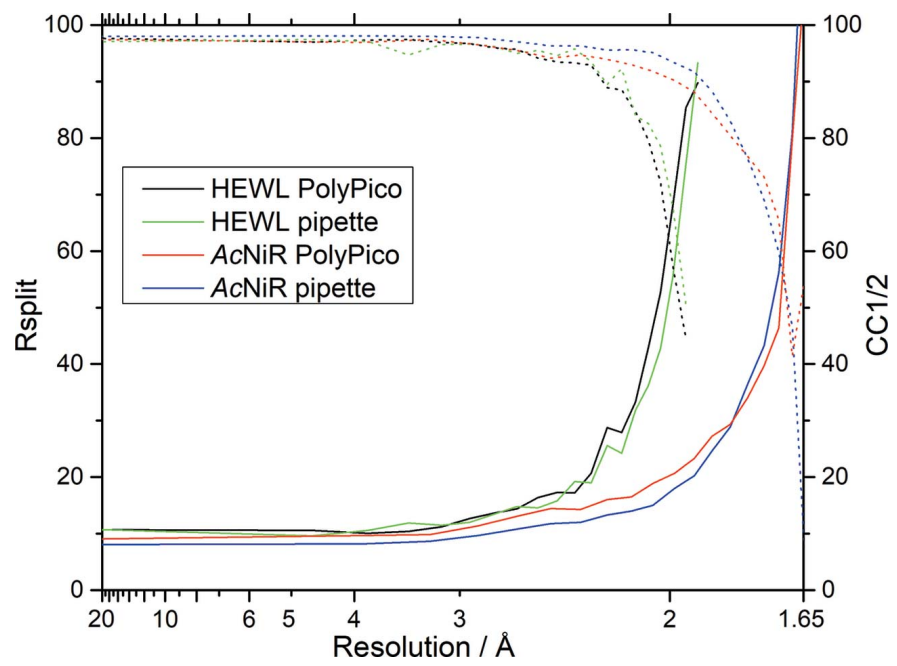

Figure 3

Data merging statistics. $R_{\text {split }}$ (solid line) and $\mathrm{CC}_{1 / 2}$ (dotted line) are shown for HEWL (black and green) and $A c \mathrm{NiR}$ (red and blue) crystals loaded on the chip with either PolyPico or a pipette. Scaling and merging have been performed using PRIME on 12546 and 13563 integrated images for HEWL and AcNiR, respectively. high. Differences in quality observed are of the same order as chip-to-chip variation when using the same loading approach, thus diffraction quality is not compromised by acoustic loading.

For both pipette and acoustically loaded chips crystals are observed to be predominantly randomly orientated on the chips, illustrated by the stereographic projections in Fig. 4. To generate these plots data were reindexed in $P 1$ so no symmetry equivalents are plotted. For more heavily loaded chips we do see some indication of systematic orientations, and this starts to become apparent in the case of pipette loaded HEWL [Fig. 4(c)]. Two orthogonal ellipses with a width of $70^{\circ}$ at the centre of the projection become visible. These are consistent with loaded crystals lying on the internal walls of the chip apertures which are chemically etched along the silicon 111 crystal planes $54.74^{\circ}$ from the surface of the chip (35.26 to the beam direction), with the ellipses reminiscent of stereographic projections of silicon etch planes as illustrated by Seidel et al. (1990). The degree of observed systematic orientation is likely to be dependent on the density of sample on the chip, crystal size and morphology, and also loading method, with acoustic loading less likely to yield systematic orientations.

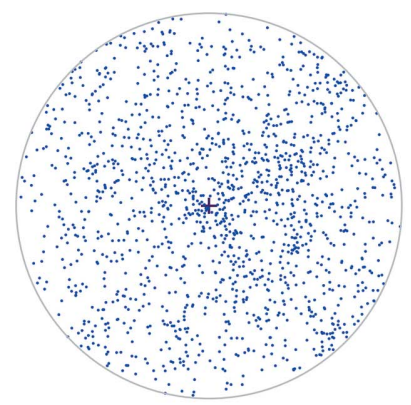

(a)

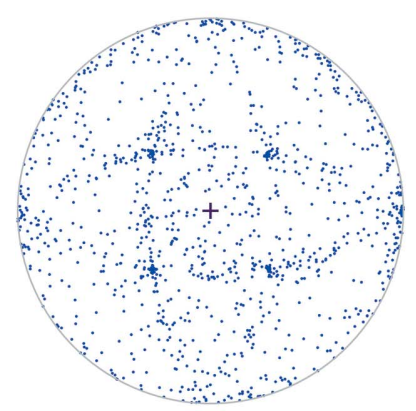

(c)

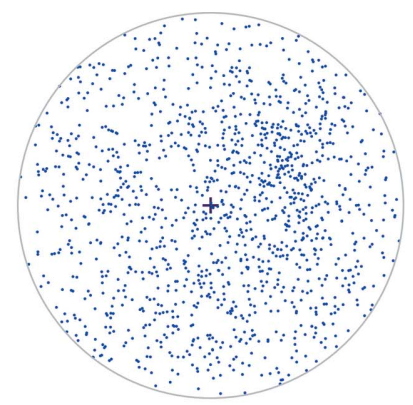

(b)

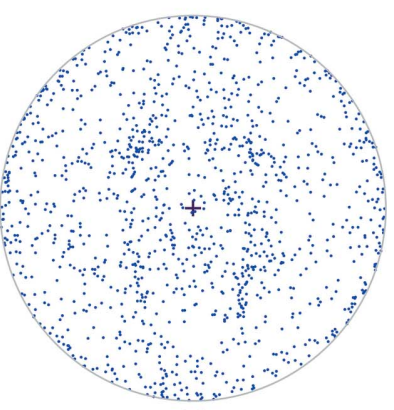

(d)
Figure 4

Stereographic projections illustrating the crystal orientation of 1000 randomly selected crystals for each loading method and crystal type: $(a)$ pipette-loaded $A c \mathrm{NiR},(b)$ PolyPico-loaded $A c \mathrm{NiR},(c)$ pipette-loaded HEWL and $(d)$ PolyPico-loaded HEWL. The plots represent the direction of the $001 \mathrm{hkl}$ of each crystal (reindexed in $P 1$ ) relative to the beam direction $(z)$ which is shown as the central ' + ' into the page. A point at 12 o'clock on the circular projection represents a $90^{\circ}$ rotation of the crystal around $x$ whereas the point at 3 o'clock represents a $90^{\circ}$ rotation around $y$. Plots were produced using the module dials.stereographic projection (Winter et al., 2018). 


\section{Discussion}

Acoustic dispensing provides a means of reducing sample consumption for serial crystallography without compromising crystal quality with high-quality diffraction observed using both loading approaches. Acoustic dispensing has been previously exploited in the context of sample delivery, whereas here it is used for loading fixed targets that are subsequently passed to the beamline. This decoupling of acoustic ejection and X-ray data collection is advantageous as time taken to optimize drop ejection, which varies with the composition of the crystal slurry, does not impact the beam time efficiency.

Optimal loading is obtained with crystals less than $\sim 50 \mu \mathrm{m}$ in size using cartridges with a $100 \mu \mathrm{m}$ aperture. Increased hitrates are obtained as the crystal slurry concentration increases, though settling of larger crystals or clumping can cause the PolyPico aperture to clog with time. This may explain why diffraction hit-rates do not increase as much as expected at the highest slurry concentration (Fig. 2). At lower crystal concentrations, we observe that the ejection process visibly disturbs the crystal slurry within the cartridge, slowing any settling process and multiple chips can be loaded from the same cartridge. Any long-term crystal settling can also be addressed by removing and reinserting the cartridge to resuspend the crystal slurry. In order to minimize any potential settling for high slurry concentrations, future loading setups will either make use of a rocking system or cartridges will be fed through a capillary fed by a syringe mounted on a rocker as used by Fuller et al. (2017).

While higher diffraction hit-rates can be obtained using traditional pipette loading, this is at the expense of increased sample consumption and the proportion of prepared crystals from which diffraction data are collected (i.e. the absolute hitrate) falls. To obtain a similar number of indexed images, acoustic dispensing consumes tenfold less crystal slurry (AcNiR, Fig. 2) than traditional pipette loading at the same sample concentration. Acoustic loading has the additional benefit that an increased fraction of the crystals produced for, and consumed by, the experiment result in diffraction. The success of acoustic loading is dependent on the chemical composition and viscosity of the crystal slurry and the parameters of the acoustic wave need to be optimized for each sample. As more viscous media may not be suitable for acoustic dispensing and the effect of crystal morphology is as yet unclear, acoustic loading of fixed targets is very much a complementary technique to pipette loading. We have demonstrated, however, that if samples are scarce, acoustic loading can help ensure a larger fraction of crystals see the $\mathrm{X}$-ray beam and reduce the volume of sample required.

\section{Funding information}

Diffraction data were measured on beamline I24 at Diamond Light Source (proposal Nos. NT14493 and MX19458). This work was supported by Diamond Light Source, which receives funding from the UK Government and the Wellcome Trust.
$\mathrm{PA}, \mathrm{AB}$ and $\mathrm{AMO}$ were also partially supported by funding from the Biotechnology and Biological Sciences Research Council and a Strategic Award from the Wellcome Trust (102593); AMO was also partially supported by a Royal Society Wolfson Fellowship (RSWF|R21182017) and a Wellcome Investigator Award in Science (210734/Z/18/Z). GL is Founder, Director and CTO of PolyPico Technologies Ltd (Cork, Ireland).

\section{References}

Brewster, A. S., Waterman, D., Parkhurst, J. M., Gildea, R. J., MichelsClark, T., Young, I. D., Bernstein, H. J., Winter, G., Evans, G. \& Sauter, N. K. (2016). Comput. Crystallogr. Newsl. 7, 32-53.

Brewster, A. S., Waterman, D. G., Parkhurst, J. M., Gildea, R. J., Young, I. D., O'Riordan, L. J., Yano, J., Winter, G., Evans, G. \& Sauter, N. K. (2018). Acta Cryst. D74, 877-894.

Demirci, U. \& Montesano, G. (2007). Lab Chip, 7, 1139-1145.

Diederichs, K. \& Wang, M. (2017). Protein Crystallography: Methods and Protocols, edited by A. Wlodawer, Z. Dauter \& M. Jaskolski, pp. 239-272. New York: Springer.

Doak, R. B., Nass Kovacs, G., Gorel, A., Foucar, L., Barends, T. R. M., Grünbein, M. L., Hilpert, M., Kloos, M., Roome, C. M., Shoeman, R. L., Stricker, M., Tono, K., You, D., Ueda, K., Sherrell, D. A., Owen, R. L. \& Schlichting, I. (2018). Acta Cryst. D74, 10001007.

Ebrahim, A., Appleby, M. V., Axford, D., Beale, J., Moreno-Chicano, T., Sherrell, D. A., Strange, R. W., Hough, M. A. \& Owen, R. L. (2019). Acta Cryst. D75, 151-159.

Fuller, F. D., Gul, S., Chatterjee, R., Burgie, E. S., Young, I. D., Lebrette, H., Srinivas, V., Brewster, A. S., Michels-Clark, T., Clinger, J. A., Andi, B., Ibrahim, M., Pastor, E., de Lichtenberg, C., Hussein, R., Pollock, C. J., Zhang, M., Stan, C. A., Kroll, T., Fransson, T., Weninger, C., Kubin, M., Aller, P., Lassalle, L., Bräuer, P., Miller, M. D., Amin, M., Koroidov, S., Roessler, C. G., Allaire, M., Sierra, R. G., Docker, P. T., Glownia, J. M., Nelson, S., Koglin, J. E., Zhu, D., Chollet, M., Song, S., Lemke, H., Liang, M., Sokaras, D., Alonso-Mori, R., Zouni, A., Messinger, J., Bergmann, U., Boal, A. K., Bollinger, J. M. Jr, Krebs, C., Högbom, M., Phillips, G. N. Jr, Vierstra, R. D., Sauter, N. K., Orville, A. M., Kern, J., Yachandra, V. K. \& Yano, J. (2017). Nat. Methods, 14, 443-449.

Grünbein, M. L. \& Nass Kovacs, G. (2019). Acta Cryst. D75, 178191.

Leen, G. (2016). Biosens. J. 5, 139.

Martiel, I., Müller-Werkmeister, H. M. \& Cohen, A. E. (2019). Acta Cryst. D75, 160-177.

Monteiro, D. C. F., Vakili, M., Harich, J., Sztucki, M., Meier, S. M., Horrell, S., Josts, I. \& Trebbin, M. (2019). J. Synchrotron Rad. 26, 406-412.

Owen, R. L., Axford, D., Sherrell, D. A., Kuo, A., Ernst, O. P., Schulz, E. C., Miller, R. J. D. \& Mueller-Werkmeister, H. M. (2017). Acta Cryst. D73, 373-378.

Roessler, C. G., Agarwal, R., Allaire, M., Alonso-Mori, R., Andi, B., Bachega, J. F. R., Bommer, M., Brewster, A. S., Browne, M. C., Chatterjee, R., Cho, E., Cohen, A. E., Cowan, M., Datwani, S., Davidson, V. L., Defever, J., Eaton, B., Ellson, R., Feng, Y., Ghislain, L. P., Glownia, J. M., Han, G., Hattne, J., Hellmich, J., Héroux, A., Ibrahim, M., Kern, J., Kuczewski, A., Lemke, H. T., Liu, P., Majlof, L., McClintock, W. M., Myers, S., Nelsen, S., Olechno, J., Orville, A. M., Sauter, N. K., Soares, A. S., Soltis, S. M., Song, H., Stearns, R. G., Tran, R., Tsai, Y., Uervirojnangkoorn, M., Wilmot, C. M., Yachandra, V., Yano, J., Yukl, E. T., Zhu, D. \& Zouni, A. (2016). Structure, 24, 631-640. 
Schlichting, I. (2015). IUCrJ, 2, 246-255.

Seidel, H., Csepregi, L., Heuberger, A. \& Baumgartel, H. (1990). J. Electrochem. Soc. 137, 3612-3626.

Sherrell, D. A., Foster, A. J., Hudson, L., Nutter, B., O'Hea, J., Nelson, S., Paré-Labrosse, O., Oghbaey, S., Miller, R. J. D. \& Owen, R. L. (2015). J. Synchrotron Rad. 22, 1372-1378.

Soares, A. S., Engel, M. A., Stearns, R., Datwani, S., Olechno, J., Ellson, R., Skinner, J. M., Allaire, M. \& Orville, A. M. (2011). Biochemistry, 50, 4399-4401.
Teplitsky, E., Joshi, K., Ericson, D. L., Scalia, A., Mullen, J. D., Sweet, R. M. \& Soares, A. S. (2015). J. Struct. Biol. 191, 49-58.

Uervirojnangkoorn, M., Zeldin, O. B., Lyubimov, A. Y., Hattne, J., Brewster, A. S., Sauter, N. K., Brunger, A. T. \& Weis, W. I. (2015). eLife, 4, e05421.

Winter, G., Waterman, D. G., Parkhurst, J. M., Brewster, A. S., Gildea, R. J., Gerstel, M., Fuentes-Montero, L., Vollmar, M., MichelsClark, T., Young, I. D., Sauter, N. K. \& Evans, G. (2018). Acta Cryst. D74, 85-97. 